\title{
Combinatorial synthesis and high-throughput photopotential and photocurrent screening of mixed-metal oxides for photoelectrochemical water splitting
}

\author{
Jordan E. Katz, Todd R. Gingrich, Elizabeth A. Santori and Nathan S. Lewis* \\ Received 16th July 2008, Accepted 28th August 2008 \\ First published as an Advance Article on the web 6th November 2008 \\ DOI: $10.1039 / \mathrm{b812177j}$
}

\begin{abstract}
A high-throughput method has been developed using a commercial piezoelectric inkjet printer for synthesis and characterization of mixed-metal oxide photoelectrode materials for water splitting. The printer was used to deposit metal nitrate solutions onto a conductive glass substrate. The deposited metal nitrate solutions were then pyrolyzed to yield mixed-metal oxides that contained up to eight distinct metals. The stoichiometry of the metal oxides was controlled quantitatively, allowing for the creation of vast libraries of novel materials. Automated methods were developed to measure the opencircuit potentials $\left(E_{\mathrm{oc}}\right)$, short-circuit photocurrent densities $\left(J_{\mathrm{sc}}\right)$, and current density $v s$. applied potential $(J-E)$ behavior under visible light irradiation. The high-throughput measurement of $E_{\mathrm{oc}}$ is particularly significant because open-circuit potential measurements allow the interfacial energetics to be probed regardless of whether the band edges of the materials of concern are above, close to, or below the values needed to sustain water electrolysis under standard conditions. The $E_{\mathrm{oc}}$ measurements allow high-throughput compilation of a suite of data that can be associated with the composition of the various materials in the library, to thereby aid in the development of additional screens and to form a basis for development of theoretical guidance in the prediction of additional potentially promising photoelectrode compositions.
\end{abstract}

\section{Introduction}

Several wide band-gap metal oxide semiconductors, such as $\mathrm{TiO}_{2}, \mathrm{SrTiO}_{3}$, and $\mathrm{KTaO}_{3}$, have been shown to split water effi-

Beckman Institute and Kavli Nanoscience Institute, Division of Chemistry and Chemical Engineering, 210 Noyes Laboratory, 127-72 California Institute of Technology, Pasadena, California 91125, USA ciently and to be chemically stable. ${ }^{1-5}$ However, these materials have band gaps of $3 \mathrm{eV}$ or larger, and hence have relatively poor overlap with the solar spectrum at the surface of the earth. This poor spectral overlap results in these materials having overall solar energy-to-fuels conversion efficiencies of $1-2 \%$. Other materials, such as CdTe or InP, have band-gaps that are better matched to the solar spectrum, but these semiconductors either corrode or develop electrically insulating overlayers when used as

\section{Broader context}

Although sunlight provides by far the most abundant renewable energy resource, solar energy utilization at massive scale will require integrated storage and distribution, in addition to technologies that enable solar energy capture and conversion. Due to their very high energy density, far dwarfing that of batteries, compressed air energy storage systems, or pumped hydro systems, chemical fuels present an extraordinarily attractive option for enabling persistent, long term, high deliverability and high energy density, solar energy storage on a massive scale. Photovoltaics in series with electrolysis units accomplish this functionality, but require expensive electrical interconnections between the two separate (individually and collectively expensive) components of the energy system. Photosynthesis accomplishes the conversion of sunlight into chemical fuel (initially in the form of NADH and ultimately in the form of reduction of $\mathrm{CO}_{2}$ to form sugars), albeit at very low $(<1 \%)$ yearly averaged real energy efficiencies. Artificial photosynthesis has been shown to produce fuel from sunlight with much higher $(>10 \%)$ overall energy conversion efficiency. However, photoelectrode materials for artificial photosynthesis that exhibit long-term stability in sunlight are relatively inefficient at fuel production, and conversely photoelectrode materials that exhibit high energy conversion efficiencies are relatively unstable. New materials that simultaneously possess both of these attributes are thus desirable. One approach to this problem, as described herein, is to explore a wide set of new photoelectrode materials in a combinatorial fashion, enabling screening of a wide variety of possibilities while compiling a structure-function database to guide yet further exploration and screening of promising new photoelectrodes. If a suitable material were successfully identified and developed, in principle it would underpin the development of a complete solar energy capture, conversion, and storage system that would be capable, if suitably widely deployed, of providing hundreds of exaJ per year of energy from sunlight in a storable, transportable, readily utilizable form, i.e. as chemical fuel. 
photoelectrodes in aqueous solution. ${ }^{6-8}$ Still other materials are stable and have band gaps relatively well matched to the solar spectrum, but do not produce photoexcited electrons with sufficient potential to reduce water to $\mathrm{H}_{2}$ and/or photogenerated holes with sufficient potential to oxidize water to $\mathrm{O}_{2}{ }^{2}$

One approach to expanding the set of suitable photoelectrode materials is to take a material that partially satisfies the spectral overlap, energetic, and stability requirements for water photoelectrolysis and attempt modify the material so as to ameliorate its deficiencies. For example, the addition of $\mathrm{Ni}$ to $\mathrm{InTaO}_{4}$ enhances the long-wavelength response of the material, producing photoactivity at wavelengths of light as long as 420 $\mathrm{nm}$, although overall energy-conversion efficiencies in sunlight are still $<1 \%$. ${ }^{9}$ Similarly, many researchers have tried to develop chemical methods to passivate the semiconductor to prevent corrosion or degradation. ${ }^{10-12}$

Another approach involves the use of combinatorial methods to synthesize new materials and characterize their photoelectrochemical properties using a high-throughput process. ${ }^{13-17}$ We present herein a high-throughput synthesis and screening methodology for testing the photoelectrochemical properties of novel semiconducting compounds. A commercial piezoelectric inkjet printer is used to quantitatively combine up to eight different metal solutions at once, and the resulting mixture is then pyrolyzed to form mixed-metal oxides. The photoelectrochemical properties of these compounds are then determined in a high-throughput fashion by measurement of the open-circuit potential, $E_{\mathrm{oc}}$, as well as the photocurrent. Measurement of $E_{\mathrm{oc}}$ under high-intensity illumination provides a measurement of the majority-carrier quasiFermi level under illumination, allowing compilation of a database of key photoelectrode performance properties associated with the materials of concern. In this fashion, the data can be used to guide secondary screens, to help formulate directions for new primary screens, and to form a basis for analysis by theory to guide exploration of additional sets of materials for desirable activity in photoelectrochemical solar-based water splitting.

\section{Experimental}

\section{A. Materials}

Glass coated with fluorine-doped tin oxide (FTO) (Hartford Glass, TEC 15) was used as the substrate for the mixed-metal

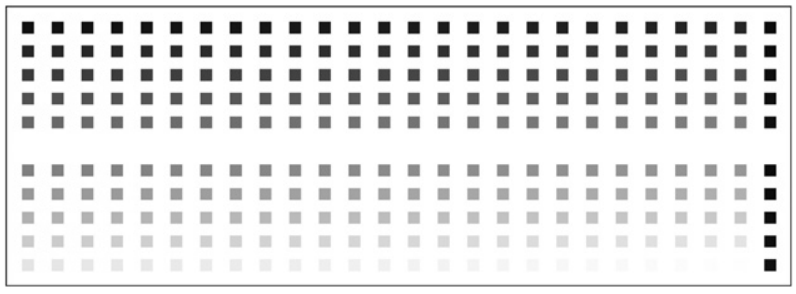

Fig. 1 The gray-scale TIFF image printed using the QTR software to make quantitatively predetermined mixtures of 255 different mixed-metal oxides on a single substrate. Rather than printing different images to generate different mixtures, the same TIFF file was used each time, but the QTR software used a new quad file to generate the desired variation in elemental composition. The right-most column is composed of five controls that are five well-characterized materials with known opencircuit potentials. oxides. Prior to deposition of the metal solutions, the FTOcoated slides were washed thoroughly with soap and water, rinsed with distilled water and isopropanol, and dried under a stream of $\mathrm{N}_{2}(g)$. The FTO was then treated with SurfaSil Siliconizing Solution (Pierce) to make the surface hydrophobic, and rinsed sequentially with isopropanol, water, and again with isopropanol, before being dried under a stream of $\mathrm{N}_{2}(g)$. The metal nitrates $\mathrm{Al}\left(\mathrm{NO}_{3}\right)_{3}, \mathrm{Cu}\left(\mathrm{NO}_{3}\right)_{2}, \mathrm{Co}\left(\mathrm{NO}_{3}\right)_{2}, \mathrm{Ni}\left(\mathrm{NO}_{3}\right)_{2}$, $\mathrm{Sr}\left(\mathrm{NO}_{3}\right)_{2}, \mathrm{Cr}\left(\mathrm{NO}_{3}\right)_{3}, \mathrm{Fe}\left(\mathrm{NO}_{3}\right)_{3}$, and $\mathrm{Zn}\left(\mathrm{NO}_{3}\right)_{2}$ (all from Aldrich and $\geq 98 \%$ purity), and a water-stable titanium complex, titanium(IV) bis(ethyl aceto-acetato)diisopropoxide (Tyzor LA, Aldrich), were used as received. The metal solutions for inkjet printing were made by dissolving sufficient metal salt into a base stock ink solution (MIS Associates) to produce $0.5 \mathrm{M}$ metal ion concentrations. To improve the solubility of some of the metal (a)

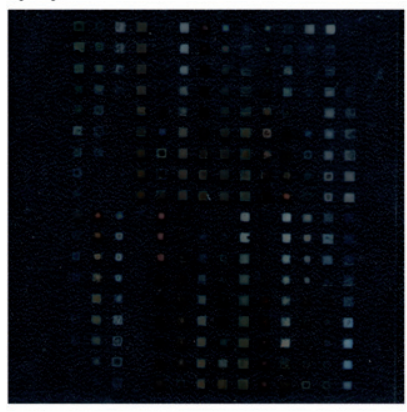

(b)

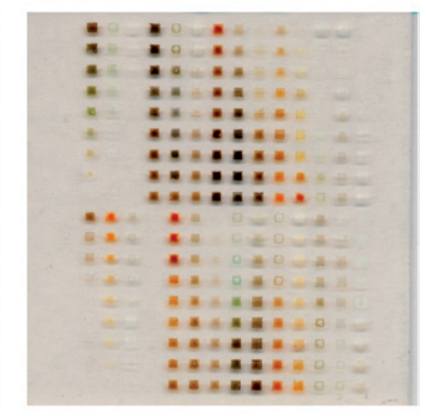

(c)

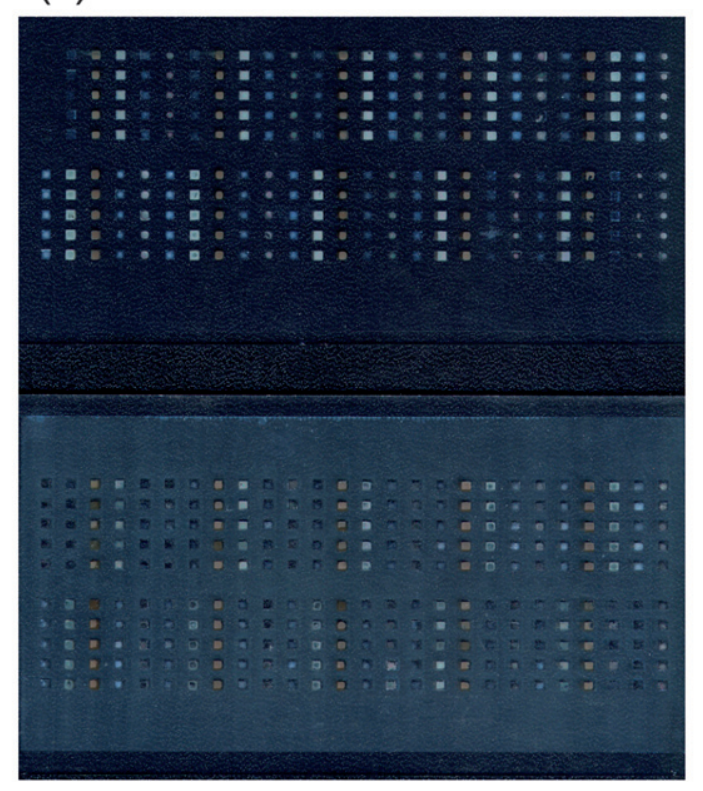

Fig. 2 Image of mixed-metal oxide spots printed on a FTO-coated glass substrate using an inkjet printer, which produced quantitatively predetermined mixtures of aqueous metal solutions that were then baked at $500{ }^{\circ} \mathrm{C}$ to form oxides. (a) and (b) Printed slides on a continuous FTOcoated glass substrate, as used for the photocurrent measurement setup. Panels (a) and (b) are images of the same slide, but a different background, for improved contrast in each image. (c) Image of the slide with a repeating pattern of five mixed-metal oxides, as described in the text. The upper image shows the slide after pyrolysis, and the bottom shows the slide after the FTO back-contact surface area had been coated with epoxy. 
salts, the base stock was first acidified with glacial acetic acid (Aldrich, > 99.99\%) to $\mathrm{pH} 4.5$ for metal nitrate solutions and to pH 7.0 for Tyzor solutions. Electrolyte solutions for photoelectrochemical characterization consisted of $1.0 \mathrm{M} \mathrm{KOH}(a q)$ in $>18.1 \mathrm{M} \Omega \mathrm{cm}$ resistivity $\mathrm{H}_{2} \mathrm{O}$, obtained from a Barnsted Nanopure system.

\section{B. Combinatorial metal oxide synthesis}

A refurbished Epson Stylus Photo R800 piezoelectric inkjet printer (Epson.com) was used to deposit mixed-metal nitrate solutions directly onto a FTO-coated glass substrate. Instead of the standard eight ink cartridges, eight empty spongeless ink cartridges (MIS Associates) were each filled with a metal ion solution. A $2.3 \mathrm{~mm}$ thick high-density polyethylene (HDPE) template (Small Parts, Inc.) was cut to allow glass slides to be fed into the front of the printer using the CD-printing functionality.

The QuadTone Raster Image Processor software (QTR) (www.quadtonerip.com) was used to obtain quantitative control of the relative proportion of the printed metal solutions. QTR allows for user-defined control of how much ink is deposited from each cartridge. Using the QTR software, a file (a so-called "quad file") was used to define each gray-scale color so as to produce a specific volume of metal solution to be printed from each printer cartridge. A gray-scale tagged image file format (TIFF) image, with a maximum of 256 distinct gray-scale colors, was then printed (see Fig. 1). The same TIFF image was printed in each case, but each time using a different quad file in QTR, resulting in a different mapping of the gray-scale color to the printed mixed-metal composition. In this way, a unique set of mixed-metal oxides was printed on each FTO-coated glass slide.

After being printed, the metal solutions were dried at $80^{\circ} \mathrm{C}$ for $\sim 30 \mathrm{~min}$, heated under flowing air at $500{ }^{\circ} \mathrm{C}$ for $3 \mathrm{~h}$, and then cooled to room temperature over $\sim 8 \mathrm{~h}$.

\section{Characterization of printed metal oxides}

To ensure quantitative mixing of the aqueous metal solutions, the composition of mixed-metal oxide samples was characterized by energy dispersive spectroscopy (EDS) using a LEO 1550VP scanning electron microscope (SEM) equipped with an Oxford INCA 300 spectrometer. After pyrolysis, the printed thickness of the metal oxide samples was determined using a Dektak 3030 profilometer.

\section{Photoelectrochemical measurements}

For photoelectrochemical measurements, a FTO-coated glass substrate was submerged in $1 \mathrm{M} \mathrm{KOH}(a q)$ (except for $1-2 \mathrm{~cm}$ at the top where electrical contact was made) and illuminated through a quartz window (GM Associates) by a $150 \mathrm{~W}$ Xe arc lamp. All measurements were made using LabView software, via a National Instruments BNC-2100 connector block and a PCI-6034E data acquisition card. The illumination intensity was measured with a Newport 815 power meter. As described below, the photoelectrochemical properties of the mixed-metal oxide materials were determined using either a photocurrentonly measurement system or using a system equipped to measure both the open-circuit photopotential and the short-circuit photocurrent.

1. Photocurrent measurements. To measure photocurrents, 252 unique metal oxides were printed on a $76 \mathrm{~mm}$ x $102 \mathrm{~mm}$ rectangular FTO-coated glass slide. Typical printed slides of mixed metal oxides are shown in Fig. 2. In this configuration, a single electrical lead made contact to all of the metal oxides on a given FTO-coated glass slide, hence each material was in electrical contact with the other materials on the slide through the continuous, conductive FTO substrate. To address an individual metal oxide region, the output of the arc lamp was focused, using quartz optics, to a spot $\sim 3-4 \mathrm{~mm}$ in diameter (Fig. 3). The beam

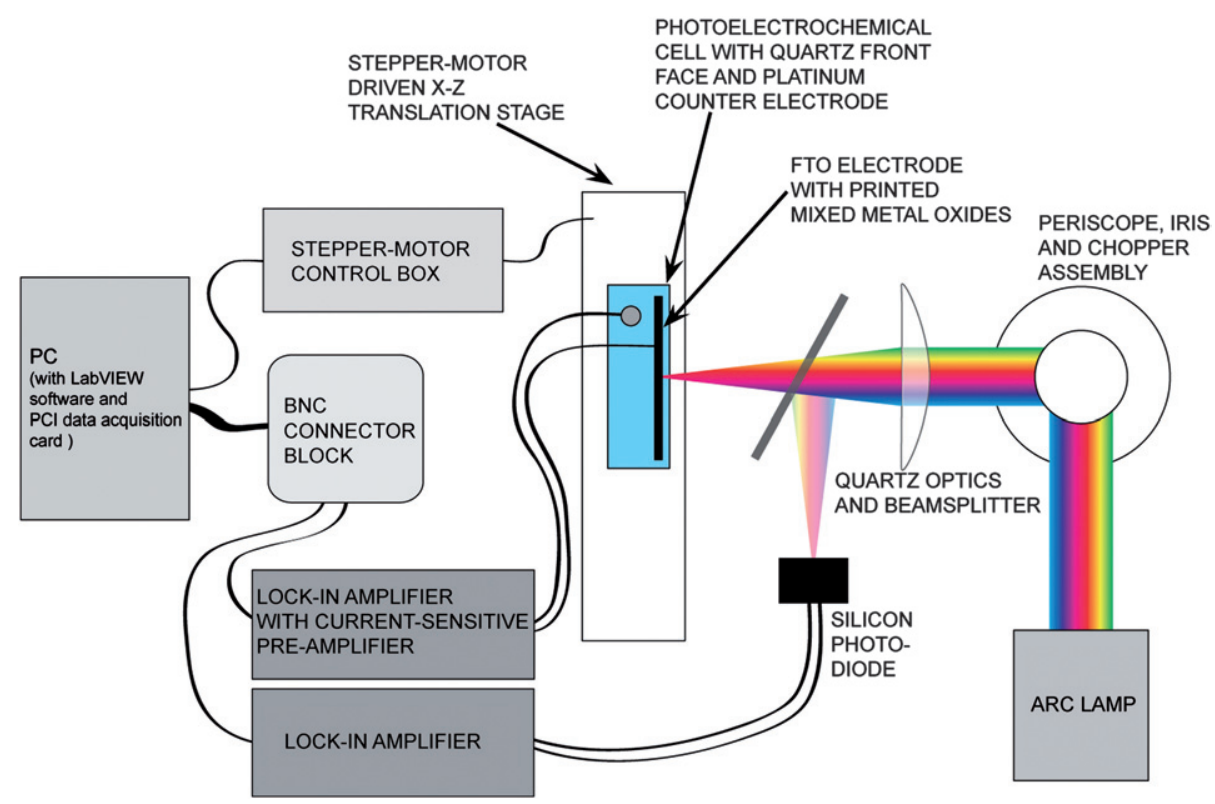

Fig. 3 Schematic of the photocurrent apparatus. A Xe arc lamp provided white light illumination that was focused onto the substrate. The entire electrochemical cell was moved using a $\mathrm{X}-\mathrm{Z}$ translation stage, illuminating in sequence each printed material on the slide. The photocurrent from the mixed-metals oxides, and from a reference Si diode, respectively, were monitored using lock-in amplifiers, and a computer then recorded both values. 
(a)
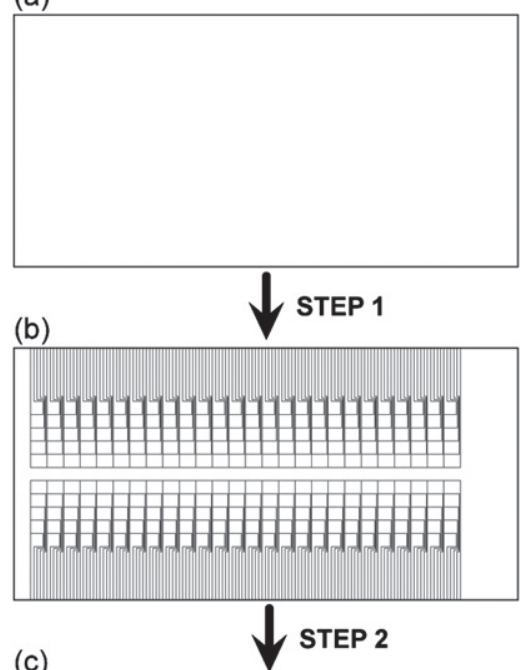

(c)

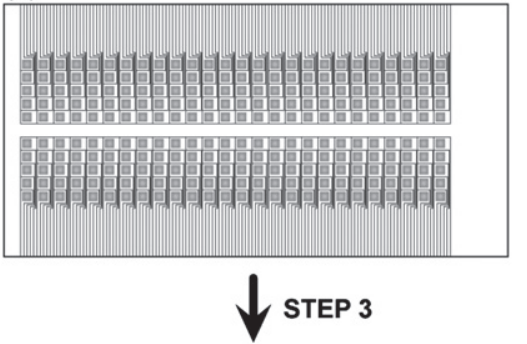

(d)
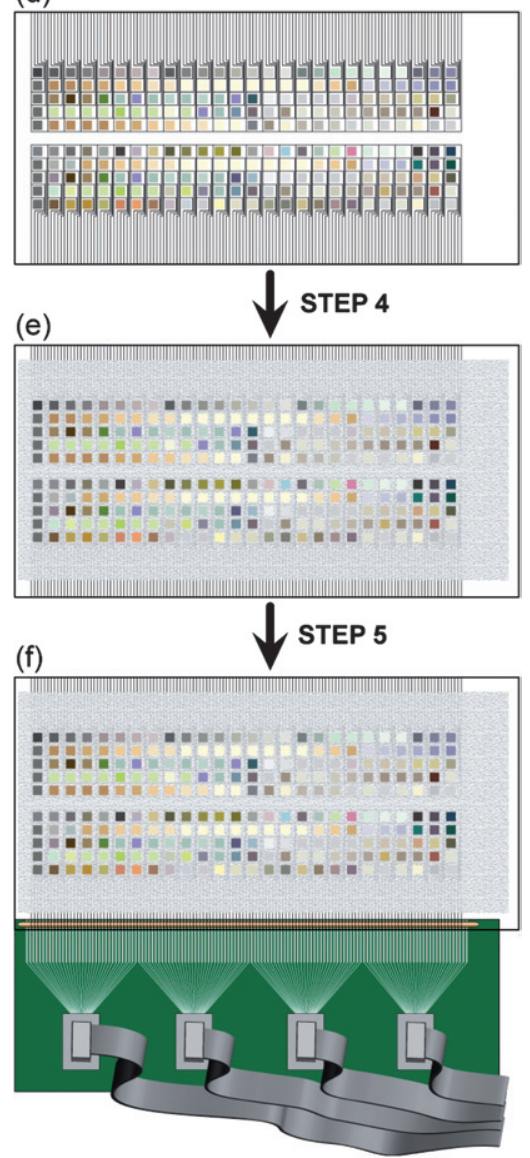

Fig. 4 Schematic of the process of preparing the substrate and making electrical contact to 130 individual materials on a single piece of FTO-coated glass. (a) The substrate started as a sheet of glass uniformly coated with FTO. (b) In Step 1, the FTO-coated glass was laser etched with a spot size of 50 $\mu \mathrm{m}$. The etched FTO substrate, with 260 electronically isolated squares, with 130 contact pads along each of the two long sides of the substrate. (c) In Step 2, the substrate was carefully cleaned and treated with siliconizing liquid for hydrophobicity, and aqueous solutions of metals were then printed using a commercial inkjet printer. After printing, the slide was dried at $\sim 80^{\circ} \mathrm{C}$. In Step 3, the substrate was baked at $500{ }^{\circ} \mathrm{C}$ for $3 \mathrm{~h}$ and cooled overnight, pyrolyzing the metal salts. (d) After baking, mixed metal oxides of varying coloration were generated. (e) In Step 4, the substrate was screen printed with a screen-printable epoxy, to passivate the surface area of the slide that was not covered by metal oxide spots. This step reduced the interfacial capacitive charging and minimized any deleterious electron-transfer processes that might occur at the FTO-water interface. (f) In Step 5, electrical contact was made to each of the 130 contact pads along the edge of the substrate using an elastomeric connector clamped between a custom-made PCB with corresponding contact pads. Each contact pad on the PCB was routed to one of four ribbon cable connectors, which were subsequently connected to a second PCB (not shown).

was chopped at $13 \mathrm{~Hz}$ (CH-61, Boston Electronics). The photoelectrochemical cell was translated in the $\mathrm{X}-\mathrm{Z}$ directions using a computer-controlled translation stage (UniSlide translation stage, Velmex, Inc; MD-2 stepper-motor driver, Arrick Robotics; MD-2 LabView driver, TEM Consulting, LP). At each position of the translation stage, the photocurrent was measured, in a twoelectrode configuration, with a Pt gauze counter-electrode, using a lock-in amplifier (EG\&G 124A) equipped with a currentsensitive preamplifier (EG\&G 184). A quartz slide split off $\sim 10 \%$ of the intensity of the arc lamp beam, and the intensity of this beam was monitored with a Si photodiode to correct for any variation in the illumination intensity.

2. Photopotential measurements. For $E_{\mathrm{oc}}$ measurements, each metal oxide material was electronically isolated from the other materials. In addition, the FTO back contact area was passivated to prevent the measurements from being dominated by the back- contact shunt that otherwise resulted from exposure of the conducting FTO to the electrolyte solution (Fig. 4). First, the FTOcoated glass slides were laser etched $(50 \mu \mathrm{m}$ laser spot size, Laserod, Inc.) to generate 260 electronically isolated conductive pads $(4 \mathrm{~mm}$ by $3.8-5 \mathrm{~mm})$ on each $76 \times 152 \mathrm{~mm}$ FTO slide, with 130 contacts along each of the two $152 \mathrm{~mm}$ edges of the slide (Fig. 4b). After depositing and pyrolyzing the aqueous metal solutions, an insulating epoxy (1 part ADE678 and 5 parts ADE26, Nazdar) was screen-printed (using a HC-53 AMI Inc. screen printer, with a $255-40$ mesh polyester screen and $12.7 \mu \mathrm{m}$ of MX emulsion, Sefar Printing) over all of the exposed FTO to electronically passivate the regions of the FTO substrate that were not covered by the printed metal oxides (Fig. 4e). The epoxy was cured for $\sim 30$ min at $80{ }^{\circ} \mathrm{C}$, and was left for $5-7$ days before use, to fully cure. Contact to each of the $1.0 \mathrm{~mm}$ center-to-center FTO contacts along the edge of the glass substrate was made using an elastomeric connector (Silver Zebra Series 5002 Solid Support, 


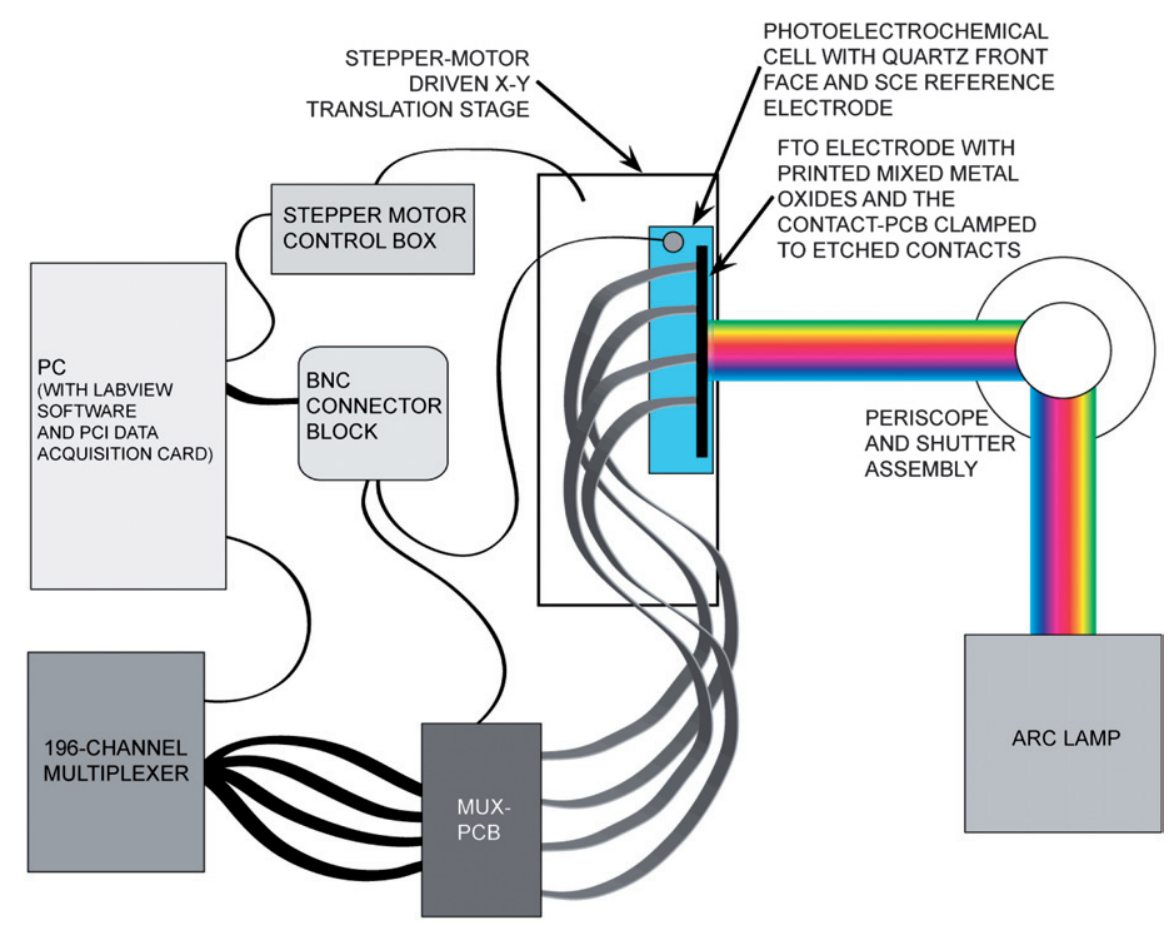

Fig. 5 Schematic of the experimental apparatus for combinatorial measurement of open-circuit potentials of mixed-metal oxides. The loosely focused output of a Xe arc lamp illuminated 10-15 printed spots at once. A motorized translation stage was used to keep the spots being measured in the region of maximum intensity as the materials on the slide were scanned from right to left. Each spot was electronically addressed individually via the contactPCB, which was clamped to the etched FTO substrate. Four 34-wire ribbon cables were routed to the mux-PCB, which was interfaced with a computercontrolled 196-channel multiplexer.

FujiPoly). The elastomeric connector was clamped, using two extended-reach clamps (McMaster Carr), between the substrate and a custom-designed printed circuit board (PCB) (Advanced Circuits), which had $1341.0 \mathrm{~mm}$ center-to-center contact pads along one edge (Fig. 4f). Each contact pad of the clamped PCB (contact-PCB) was connected via a second PCB (mux-PCB) to the four 50-pin D-subminiature connectors of a National Instruments LHF200 switch cable, which was in turn connected to a 196-channel multiplexer (SCXI-1175, National Instruments) (Fig. 5). The open-circuit potential obtained from each spot was measured vs. a saturated calomel reference electrode (SCE). To determine the effect of illumination relative to the equilibrium potential in the dark, the light beam was blocked for $\sim 1 \mathrm{~s}$, and the rise or decay in $E_{\mathrm{oc}}$ was subsequently monitored.

3. Current-density vs. potential measurements. A nearly identical setup as that described for $E_{\mathrm{oc}}$ measurements was used to measure the current-density vs. potential behavior under potentiostatic control (SI 1287, Solartron) for mixed metal oxides. Measurements were performed $v s$. a SCE at a scan rate of $20 \mathrm{mV} \mathrm{s}^{-1}$ in a three-electrode setup with a $\mathrm{Pt}$ gauze counter electrode. Measurements were made both in the dark and under illumination.

\section{Results}

\section{A. Quantitative mixing of aqueous metal ion solutions with an} inkjet printer

1. Inkjet printing. A commercially available Epson R800 inkjet printer was used to quantitatively print mixtures of aqueous metal solution on glass substrates, with minimal modifications to the printer. A built-in printer functionality (designed to allow printing on CDs and DVDs) was used to print on rigid glass substrates. A simple HDPE template replaced the $\mathrm{CD}$ tray that is used to feed a CD into the printer. The template consisted of a rectangular $(210 \mathrm{~mm} \times 300 \mathrm{~mm})$ sheet of HDPE with a hole cut out of the center corresponding to the size of the FTO-coated glass substrate. Because of the method by which the printer senses the position of the loaded template when it is loaded, it was possible to easily print on the FTO substrate with very high reproducibility and spatial resolution $( \pm 0.5 \mathrm{~mm})$.

A printer driver other than the Epson-provided driver was needed to print mixtures with pre-determined compositions. The QTR software allows the user to create a complete colormanaged system for black and white printing. To print using QTR, a single TIFF image, consisting of 256 different gray-scale colors (see Fig. 1), was generated using Matlab. In a separate "quad file," each color was uniquely defined in terms of a specific amount of solution to be dispensed from each of the eight printer cartridges. The same TIFF image was printed on each substrate, but a different quad file was selected to create 255 new mixedmetal oxides, (the 256th gray-scale color was reserved for the area surrounding the spots, and thus always defined as printing nothing).

2. Determination of metal oxide composition. The composition of a test set of the printed spots was confirmed by EDS, after pyrolysis of the aqueous metal solutions at $500{ }^{\circ} \mathrm{C}$. Although the pyrolysis temperature is clearly a critical parameter in 
Table 1 Representative values of the measured (by EDS) and expected fractional elemental composition of binary mixed-metal oxides. The expected value is given by the ratio of the volumes of metal ion solutions specified in the quad file using the QTR software. The quoted errors are absolute composition errors

\begin{tabular}{lllll}
\hline Measured & & Expected & & Error \\
\hline $\mathrm{Fe}$ & $\mathrm{Co}$ & $\mathrm{Fe}$ & $\mathrm{Co}$ & \\
0.58 & 0.42 & 0.70 & 0.30 & $12 \%$ \\
0.51 & 0.49 & 0.60 & 0.40 & $9 \%$ \\
0.41 & 0.59 & 0.50 & 0.50 & $9 \%$ \\
0.32 & 0.68 & 0.40 & 0.60 & $8 \%$ \\
0.24 & 0.76 & 0.30 & 0.70 & $6 \%$ \\
0.15 & 0.85 & 0.20 & 0.80 & $4 \%$ \\
0.06 & 0.94 & 0.10 & 0.90 & $4 \%$ \\
& & & & \\
$\mathrm{Co}$ & $\mathrm{Ni}$ & $\mathrm{Co}$ & $\mathrm{Ni}$ & \\
0.88 & 0.12 & 0.90 & 0.10 & $2 \%$ \\
0.81 & 0.19 & 0.80 & 0.20 & $1 \%$ \\
0.35 & 0.65 & 0.40 & 0.60 & $5 \%$ \\
$\mathrm{Cu}$ & & & & \\
0.31 & $\mathrm{Sr}$ & $\mathrm{Cu}$ & $\mathrm{Sr}$ & \\
0.23 & 0.69 & 0.40 & 0.60 & $9 \%$ \\
$\mathrm{Cu}$ & 0.77 & 0.30 & 0.70 & $7 \%$ \\
0.37 & & & & \\
0.47 & $\mathrm{Cr}$ & $\mathrm{Cu}$ & $\mathrm{Cr}$ & \\
0.56 & 0.63 & 0.30 & 0.70 & $7 \%$ \\
$\mathrm{Co}$ & 0.53 & 0.40 & 0.60 & $7 \%$ \\
0.41 & & 0.50 & 0.50 & $7 \%$ \\
0.49 & 0.59 & $\mathrm{Cu}$ & $\mathrm{Cu}$ & \\
\hline
\end{tabular}

determining the crystallinity and/or phase of the resulting material, a maximum temperature of $550^{\circ} \mathrm{C}$ is set by the stability of the FTO-coated glass. ${ }^{13}$ A set of $\sim 100$ spots was tested, with the concentration of each metal in each mixture determined by the EDS INCA software, which calculates atomic percent compositions based on the acquired spectrum. Table 1 presents typical results from the compositional analysis of assorted metal oxide mixtures. In all cases, the elements present in the substrate were present in the EDS spectra. Only the elements specified in the quad file to be printed on a given spot were ever observed, except oxygen, silicon, tin, and calcium, which arose from the FTO-coated glass substrate.

For binary combinations of metals, the EDS analysis matched the expected percentage to within 5-10 percentage points (as much as 15 , but within 10 percentage points the large majority of the time). This accuracy should be sufficient for an initial screen of the metal oxide compositional space.

\section{B. Photoelectrochemical characterization}

1. Photocurrent measurements. In the dark, a significant and varying current was measured at short circuit. The light source was thus chopped, allowing the $\mathrm{AC}$ effect of the light to be evaluated. The polarity of the photocurrent was indicative of whether the material exhibited photocathodic or photoanodic behavior. The photocurrent was measured for each spot for $\sim 5 \mathrm{~s}$, or until a stable value was obtained. Fig. 6 shows a false-color image of the photocurrent as a function of location on the FTOcoated glass substrate.

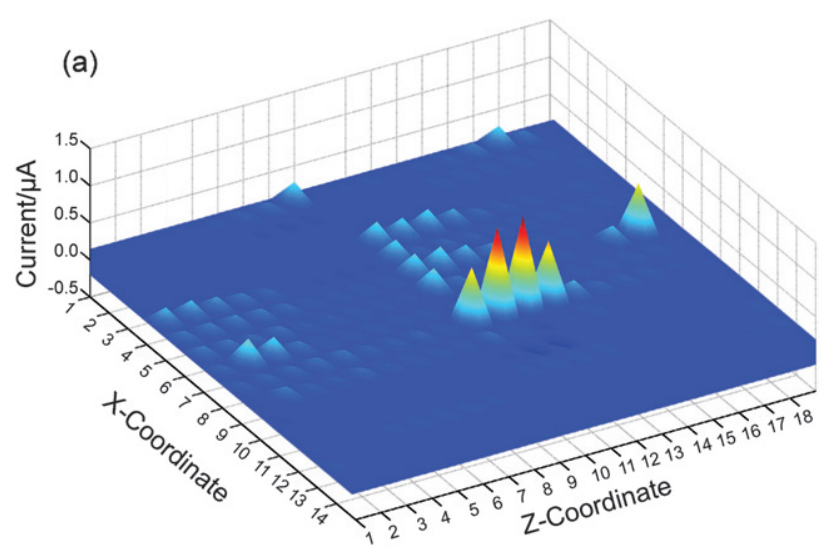

(b)

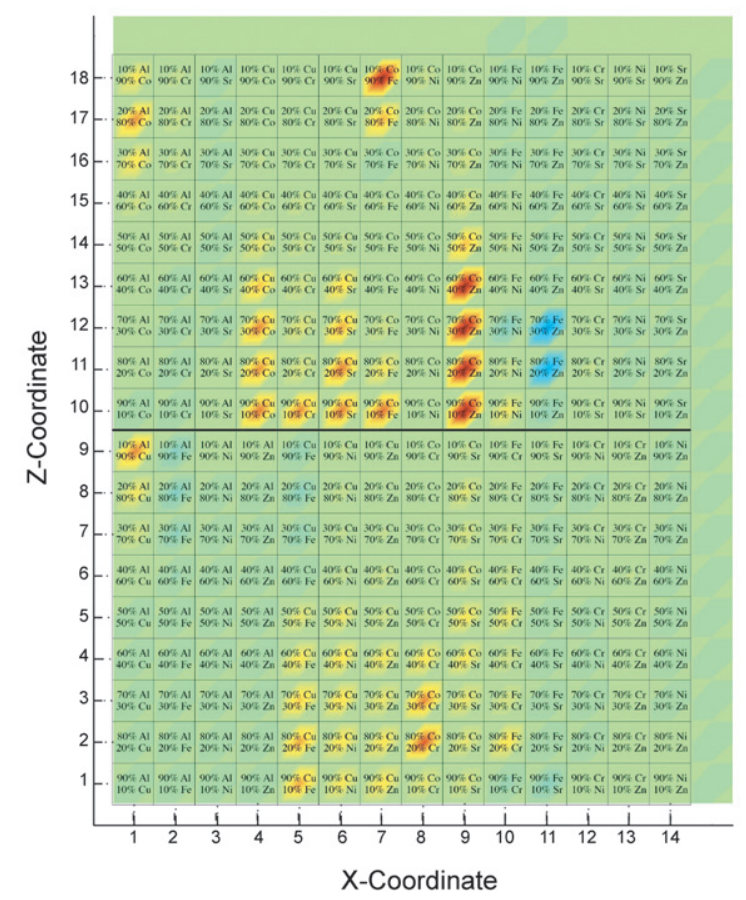

Fig. 6 False color images of the photocurrent of a slide that contained spots of binary mixed-metal oxides. (a) Three-dimensional plot showing the physical position of each compound on the FTO substrate (X- and Zcoordinates correspond to the row and column of the spot, respectively) and the photocurrent measured for each spot. Positive values indicate a cathodic photocurrent. Column 15 and row 19 had nothing printed on them, and the bare FTO served as a control. (b) Top-down view of the same data, with the composition of each spot superimposed on a falsecolor image indicating its photocurrent. In this view, the materials that gave anodic currents are more clearly visible (light blue spots-in particular in rows $11-12$, column 11).

Initial measurements revealed a number of metal oxides that exhibited anodic or cathodic photocurrents having a magnitude between $10^{-9}$ and $10^{-6} \mathrm{~A}$ in the probed $\sim 4 \mathrm{~mm}^{2}$ printed spot area. While most materials tested gave no photoresponse at all, several binary combinations gave particularly strong photocathodic currents, including combinations of $\mathrm{Co}$ and $\mathrm{Zn}$ (80-20 and 70$30 \% \mathrm{Co}$ and $\mathrm{Zn}$, respectively), $\mathrm{Co}$ and $\mathrm{Fe}(90 \% \mathrm{Co}$ and $10 \% \mathrm{Fe})$, and $\mathrm{Al}$ and $\mathrm{Co}(80 \% \mathrm{Co}$ and $20 \% \mathrm{Al})$ (Fig. 6). For many 
materials, de-oxygenation of the electrolyte solution, by bubbling $\mathrm{N}_{2}(g)$ through the cell, resulted in significantly increased photocurrents. Additionally, for several materials, the measured photocurrent changed over time. These changes included both increases and decreases in the magnitude of the photocurrent on the order of $50-100 \%$, and occurred over a period of hours as well as from day to day.

2. Open-circuit potential measurements. Initially, the $E_{\mathrm{oc}}$ of the mixed-metal oxides was measured in essentially the same way as the photocurrent. In this approach, illumination of individual materials on the slide was used instead of electronic isolation, but the light source was not chopped. However, even for well-characterized materials, the $E_{\mathrm{oc}}$ values measured in this fashion were significantly more positive than expected. For example, for $\mathrm{TiO}_{2}$, a potential of $-0.15 \mathrm{~V} v s$. SCE was observed, but at $\mathrm{pH} 14, \mathrm{TiO}_{2}$ has a flat-band potential of $-1.1 \mathrm{~V} v s$. SCE. ${ }^{18}$ In addition, photoactive materials required as long as 30 min under illumination to exhibit a stable value of $E_{\mathrm{oc}}$. The decay back to the equilibrium potential in the dark was even slower, thereby requiring nearly $12 \mathrm{~h}$ to scan a single slide that contained 252 materials (as opposed to $<30 \mathrm{~min}$ for measurement of the photocurrents).

The combination of etching the FTO-coated glass substrate to electronically isolate the spots, and covering the FTO back contact area with epoxy, solved the issues with the low $E_{\mathrm{oc}}$ values and the long apparent electrode response and recovery times. To electronically contact 130 individual spots on the etched FTOcoated glass electrode, a contact PCB, with a high density of metallic contact pads along one edge (as shown in Fig. 4f) was designed. An elastomeric connector was used to improve the conductivity of the connection, resulting in negligible contact resistance between the FTO and the contact PCB. To ensure that

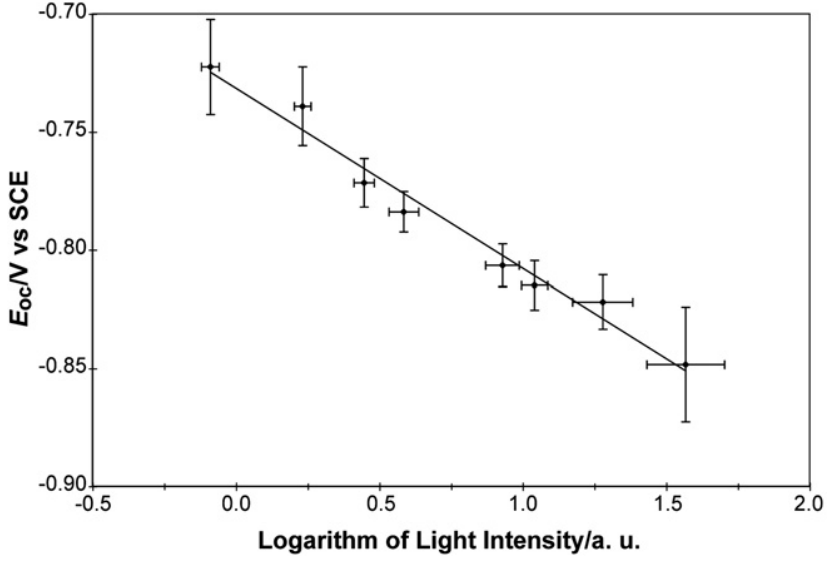

Fig. 8 Linear fit of the measured open-circuit potential $v$ s. the logarithm of the light intensity for $\mathrm{TiO}_{2}$ samples. The slope of the fit was $-76 \pm 12$ $\mathrm{mV}$ per decade. The sign of the slope indicates that $\mathrm{TiO}_{2}$ is a photoanode.

proper alignment was achieved each time, a resistance measurement was performed across two pairs of contacts on each end of the FTO-coated glass substrate.

Metal oxides printed on etched FTO substrates gave substantially faster response times, but the measured $E_{\mathrm{oc}}$ still did not match the values expected for test materials, in particular for $\mathrm{TiO}_{2}$. For $\mathrm{TiO}_{2}$ at the same illumination intensity, $E_{\mathrm{oc}}$ changed from $-0.81 \mathrm{~V}$ to $-0.88 \mathrm{~V}$ vs. SCE by simply coating with epoxy the surrounding FTO substrate that was exposed to the electrolyte. Under strong illumination, the measured $E_{\mathrm{oc}}$ of $\mathrm{TiO}_{2}$ was within $200 \mathrm{mV}$ of the reported flat-band potential for $\mathrm{TiO}_{2}$ at $\mathrm{pH}$ 14. Finally, covering the edges of the sample with transparent epoxy resulted in $E_{\mathrm{oc}}=-1.1 \mathrm{~V}$ vs. SCE for $\mathrm{TiO}_{2}$.

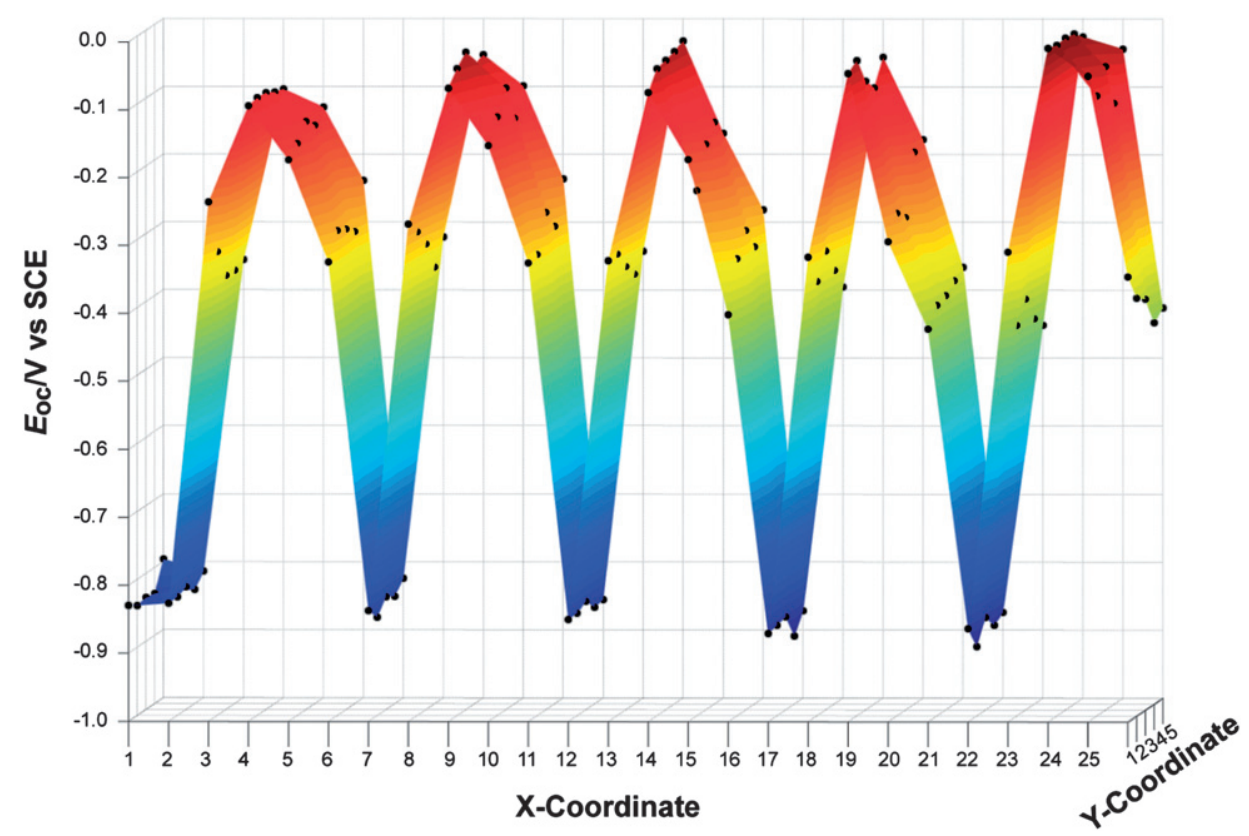

Fig. 7 Open-circuit potentials for a control sample of mixed-metal oxides as a function of position on the FTO substrate. A photograph of the substrate itself is shown in Fig. 2c. A repeating pattern of five mixed-metal oxides was printed from left to right (with one extra column of TiO ${ }_{2}$ at an $x$-coordinate equal to one). With each series of five columns (i.e., $x$-coordinates $2-6,7-11,12-16$, etc.), the volume of aqueous metal solution that was inkjet printed varied from 30 to $62 \%$ of the maximum possible, resulting in material thicknesses from $180 \mathrm{~nm}$ to $420 \mathrm{~nm}$. The open-circuit photopotentials were only weakly dependent on the relative thickness of the printed oxide, and were highly reproducible for nominally identical printed spots. 
To test the overall measurement methodology, a repeating pattern composed of five materials was generated on a single FTO substrate (Fig. 2c). Each material was printed with five thicknesses (as controlled by changing the total volume of metal solution deposited by the printer), and each material was printed five times at each thickness value. Fig. 7 shows the $E_{\text {oc }}$ values recorded from this slide. Some variation was observed in $E_{\mathrm{oc}}$ as a function of spot thickness, but spots with nominally identical compositions gave similar $E_{\mathrm{oc}}$ values.

The $E_{\mathrm{oc}}$ of the printed metal oxide materials in the dark varied greatly (values between $+0.2 \mathrm{~V}$ and $-0.3 \mathrm{~V}$ vs. SCE were observed). This variation was not unexpected, because the solution potential is not well defined in the dark, but the variation introduced ambiguity in the determination of the polarity of the photocatalytic activity. Blocking the light beam briefly and measuring the shift in $E_{\mathrm{oc}}$ from light to dark was used to verify the effect of illumination.

Fig. 8 shows a logarithmic dependence of $E_{\mathrm{oc}}$ on the illumination intensity on a printed $\mathrm{TiO}_{2}$ spot. The slope of a linear fit of $E_{\mathrm{oc}} v s$. the logarithm of the illumination power was $-76 \pm 12 \mathrm{mV}$ per decade.

3. Current density vs. potential behavior. The electronic isolation of each spot enabled combinatorial measurement of the complete current density $v s$. applied potential $(J-E)$ behavior of individual printed spots. Fig. 9 shows representative $J-E$ data for samples of printed $\mathrm{TiO}_{2}$ both in the dark and under illumination. The photocurrent of the $\mathrm{TiO}_{2}$ showed a significant dependence on the amount of ink printed, with a maximum photocurrent observed for samples with an ink level of $46 \%$ of the maximum allowed by the QTR software, corresponding to a spot thickness

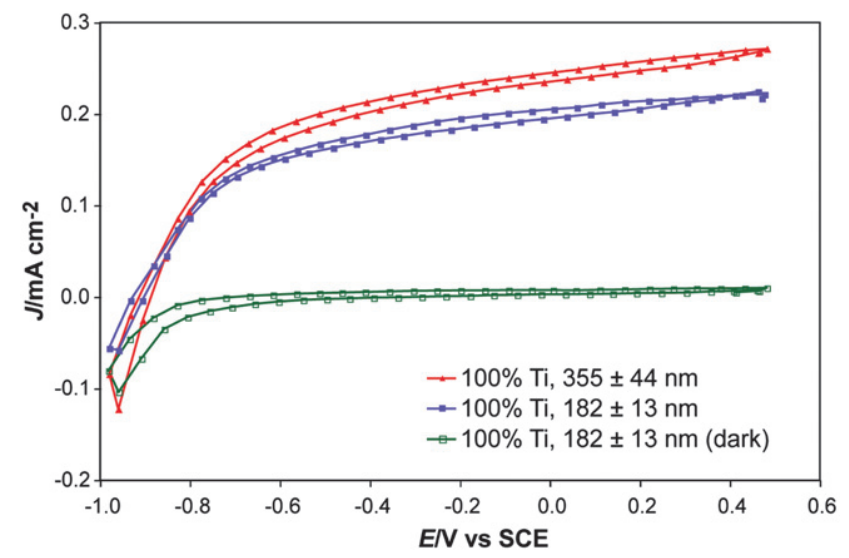

Fig. $9 J-E$ behavior of inkjet-printed $\mathrm{TiO}_{2}$ on an etched FTO-coated glass substrate. The measured open-circuit potentials were the same, within the experimental error, as those measured by scanning $J-E$ curves as well as by direct open-circuit measurements (although the $J-E$ curves show non-negligible hysteresis). These measurements illustrate the flexibility of electronically isolating each material on the substrate, and also indicate that the volume of solution printed did significantly affect the measured photocurrent, but the open-circuit potential was relatively unchanged. Although the short-circuit current is in general a linear function of illumination intensity and of the light-harvesting ability of the material, the open-circuit potential has a much less sensitive, logarithmic dependence, on the light intensity. This means that day-to-day variability in light intensity or in a given material's optimal deposition thickness will have a minor affect on the open-circuit potential screening results. of $355 \pm 44 \mathrm{~nm}$. The $E_{\mathrm{oc}}$ values measured by scanning $J-E$ measurements corresponded closely to those measured directly at open-circuit. No significant effect was observed as a function of position of the spot in a given column on the slide, despite the varying resistivity of the connecting FTO "wire" (which varied from $\sim 200$ to $\sim 1000 \Omega$ ) due to the varying distance of the spot from the elastomeric connector.

\section{Discussion}

Combinatorial synthesis and screening of novel mixed-metal oxide photoelectrodes has been demonstrated previously. ${ }^{13-17}$ The methodology described here affords several interesting capabilities. The first is the large number of metals that can be combined simultaneously during a single printing. The second is the information gleaned from an $E_{\text {oc }}$ measurement. The third is the flexibility in the types of photoelectrochemical measurements that can be made combinatorially and with high throughput.

\section{A. Inkjet printing of aqueous metal oxide precursors}

One important advantage of using the deposition method described herein is the very low cost for the entire printing system, which required no significant modification to a commercially available commodity inkjet printer. Using the QTR software, the printer provided accurate and reproducible deposition of solution volumes, allowing simultaneous control of both the spot composition and the total amount of material printed. This capability obviated the need to reprint a spot multiple times to increase the spot thickness, which in turn requires precise alignment of the substrate each time. Furthermore, the printer's eight ink cartridges allow a very large number of materials to be printed without changing the solutions in the printer, greatly decreasing the time required to print a library of mixtures consisting of three or more metals as compared to the printing time of a system that dispenses only one metal solution at time. The ability to load and use eight solutions simultaneously also minimized cross-contamination that could otherwise arise from the repeated printing of different solutions through a common set of printer nozzles.

Rather than producing a relatively smooth gradient of compositions, as reported by Woodhouse et al. ${ }^{13}$ the system described herein used a quantized step size in elemental composition of the deposited metal salts. This step size must balance the need to fully explore the eight-dimensional compositional space while limiting the number of materials to synthesize and characterize in a preliminary compositional screen. For example, to prepare every possible combination of the eight metal solutions using a composition step size of $10 \%$ by mole fraction would require 78 FTO-coated glass slides each containing spots having 255 unique materials. If a promising material were found, the compositional parameter could be further optimized in a secondary screening step, to maximize the photoactivity.

Printing isolated spots of mixed-metal oxides provided several advantages relative to printing overlapping gradients. ${ }^{13}$ For photocurrent measurements, the relatively large spot-to-spot distance meant that the spatial resolution of the illumination source was not critical to the measurement, allowing the use of the focused beam of an arc lamp to provide intense white light 
excitation, rather than a continuous wave laser. Use of white light from an arc lamp is extremely versatile, in that its broad spectrum has significant intensity from $200 \mathrm{~nm}$ to $2500 \mathrm{~nm}$. The use of a series of long-pass (or band-pass) optical filters in the beam path provides a simple, low-cost method for determining the wavelength dependence of the photoresponse of the metal oxides. Additionally, separation of the printed spots relaxes the need for high spatial resolution for other methods of characterization (beyond the scope of this study), such as XRD, EXAFS or XANES, to determine the phase, crystallinity or oxidation states of the printed materials.

\section{B. Photocurrent measurements}

The ability to generate photocurrent with a high external quantum yield (i.e., the fraction of electrons collected per incident photon) is an essential property of any efficient solar cell. However, the value of the photocurrent contains limited information as a criterion upon which to base a search for materials that can split water with visible light. Photocurrent can be generated by processes other than the desired water-splitting reaction, and conversely, a significant level of impurities in the material in the deposited spot could result in little or no photocurrent from an otherwise promising material.

In a combinatorial screening system, a significant percentage of false positives are time consuming and inconvenient, in that they prompt more detailed investigation of a material that is in the end unsatisfactory. Our efforts to screen potential materials using only the photocurrent response resulted in a large number of such false positives. Conclusive determination of the origin of the photocurrent is at present far too time-consuming to be compatible with the high-throughput combinatorial approach of interest herein. In fact, the large majority of the photocurrents shown in Fig. 6 were ultimately deemed to be due to deleterious current-producing side reactions, rather than water splitting, as evidenced by the irreproducible magnitude of the photocurrent for these samples.

In addition to the inconvenience of false positives, photocurrent measurements fail to identify "near misses." If a material that very nearly satisfied all of the energetic and stability requirements for water photoelectrolysis were synthesized, and a low photocurrent was measured, that material would be discarded and perhaps never re-investigated. For example, failure to absorb a significant amount of light due a combination of a thin printed sample and a relatively low absorption coefficient could lead to a low photocurrent. Another mechanism to produce low photocurrents is if the material absorbs light and produces electrons and holes at the required potentials for water-splitting, but has very slow interfacial electron-transfer kinetics. Yet another possibility is that perhaps the material is ideal in all ways except that the potential of the bottom of the conduction band is just very slightly more positive than the hydrogen evolution potential or the potential of the top of the valence band is very slightly more negative than the oxygen evolution potential.

\section{Open-circuit potential measurements}

1. Features of $\boldsymbol{E}_{\mathrm{oc}}$ measurements. An advantage of $E_{\mathrm{Oc}}$ measurements is that they reveal the position of the majority- carrier quasi-Fermi level of the material under illumination. This value must be more negative than the $\mathrm{H}_{2} \mathrm{O} / \mathrm{H}_{2}$ potential for a photoanode, or more positive than the $\mathrm{O}_{2} / \mathrm{OH}^{-}$potential for a photocathode, to sustain the unassisted photoelectrolysis of water under standard-state conditions. The value of the majority-carrier quasi-Fermi level thus reveals not only if a material is thermodynamically capable of effecting the relevant half-reactions for water splitting, but if not, how close it is to doing so. In addition, if the material has band edges with sufficient potential to drive both half-reactions of water electrolysis, but a catalyst is necessary for the reaction to occur at an appreciable rate, the material would still be considered noteworthy in an $E_{\mathrm{oc}}$ screen, but would likely be overlooked by screening only the photocurrent. Also, if for instance a photoanodic material had a conduction band-edge potential just slightly more positive than the $\mathrm{H}_{2} \mathrm{O} / \mathrm{H}_{2}$ potential, this would be noted and further fine-tuning of the material's composition or morphology could potentially lead to the desired band-edge position.

$E_{\mathrm{oc}}$ measurements also allow one to search for a complementary pair of photoanodes and photocathodes for water splitting. Rather than finding a single material that simultaneously satisfies all of the energetic and stability conditions for unassisted water photoelectrolysis, two light-absorbing materials can in principle be used in conjunction with each other. Such an approach changes two of the essential requirements: in a two-material case, the sum of the band-gaps of the materials must be $>1.23 \mathrm{eV}$, and the conduction band edge of the photocathode must be more negative than the $\mathrm{H}_{2} \mathrm{O} / \mathrm{H}_{2}$ potential, while the valence band edge of the photoanode must be more positive than the $\mathrm{O}_{2} / \mathrm{OH}^{-}$ potential. Thus, by using two separate materials, the restrictions on viable materials are notably relaxed. Monitoring only the photocurrent, it is not possible to search individually for two materials that could drive each half-reaction when used together, since neither material would be capable of splitting water alone when used with a metallic counter-electrode.

2. Use of $\boldsymbol{E}_{\mathrm{oc}}$ measurements to differentiate between photocathodes and photoanodes. A number of proof-of-concept experiments were performed to test the photoelectrode screening system developed herein, using both an assortment of mixed metal oxides as well as the well-characterized photoanode, $\mathrm{TiO}_{2}$. Using a kinetic model of interfacial electron transfer under illumination, the quasi-Fermi level of the material is expected to vary logarithmically with illumination intensity, by $-59 \mathrm{mV}$ per decade. ${ }^{3}$ This expectation is in accord with observations, as shown in Fig. 8. This behavior provides strong evidence that the measured $E_{\mathrm{oc}}$ value is indeed determined by the majority-carrier quasi-Fermi level under illumination. Additionally, the sign of the slope indicates that $\mathrm{TiO}_{2}$ is a photoanode, which agrees with the high-throughput method of deducing polarity from the direction of $E_{\mathrm{oc}}$ drift immediately after the light source is blocked.

Although it is important to compare $E_{\mathrm{oc}}$ at constant illumination for all samples, the logarithmic dependence ensures that $E_{\mathrm{oc}}$ varies by less than $100 \mathrm{mV}$ per order of magnitude change in intensity. ${ }^{3}$ Variations in illumination intensity from spot to spot of more than a factor of two are very unlikely given the experimental setup. Furthermore, any photoanode that produces a majority-carrier quasi-Fermi level position under illumination 
within even $100-300 \mathrm{mV}$ of the $\mathrm{H}_{2} \mathrm{O} / \mathrm{H}_{2}$ potential would prompt further investigation. Preliminary screening of some 250 mixed metal oxides revealed no materials that produced $E_{\text {oc }}$ within 0.5 $\mathrm{V}$ of the $\mathrm{H}_{2} \mathrm{O} / \mathrm{H}_{2}$ potential (except for $\mathrm{TiO}_{2}$ ), and so choosing a relatively low threshold of $E_{\mathrm{oc}}$ for screening materials may in fact be sufficiently selective for secondary study.

If $E_{\mathrm{oc}}$ alone is measured, whether the material is a photoanode or photocathode, or even photoactive at all, can be ambiguous, due to the fact that there are two redox couples in solution with which the material can interact. Whether a material is photoactive is not always trivial to determine from $E_{\mathrm{oc}}$ alone, because of the varying potential of different spots in the dark produced by the lack of a well-defined solution redox potential in electrolytes that lack a rapid one-electron redox species. A material with $E_{\mathrm{oc}}=+0.2 \mathrm{~V} v s$. SCE would be considered a very good photocathode in the screening process, but if the dark potential was already at that value due to variations in the poorly defined solution potential and not due to a photoelectrochemical effect, the screening method would have actually returned a false positive.

For a large negative $E_{\mathrm{oc}}$ value (e.g., $E_{\mathrm{oc}}<-1.1 \mathrm{~V} v s$. SCE), one might safely assume that the material is a good photoanode and that the majority-carrier quasi-Fermi level is negative of the $\mathrm{H}_{2} \mathrm{O} / \mathrm{H}_{2}$ potential. Similarly, a large positive $E_{\mathrm{oc}}$ value (e.g., $E_{\mathrm{oc}}$ $>0.2 \mathrm{~V} v s$. SCE) would indicate a good photocathode, with the majority-carrier quasi-Fermi level positive of the $\mathrm{O}_{2} / \mathrm{OH}^{-}$ potential. Intermediate $E_{\mathrm{oc}}$ values are harder to interpret without further information. A material with $E_{\mathrm{oc}}=0 \mathrm{vs}$. SCE might be selected as a reasonable candidate for further investigation if it was a photocathode. In contrast, a photoanode with such a positive $E_{\mathrm{oc}}$ would be considered a very unsuitable material for water splitting. A third possible explanation is that the material could be inactive but the Nerstian potential of the cell in the dark happened to equal the potential of a SCE.

The sign of the slope of $E_{\mathrm{oc}} v s$. the logarithm of the illumination intensity determines whether the material is photoanodic or photocathodic. However, making measurements for a full series of light intensities for each material is too time-consuming for a high-throughput approach. The sign of the slope is what is essential here, not the exact value of the slope itself, and so the simplest possible comparison of light intensities can be made quickly and easily: light $v s$. dark.

The change in $E_{\mathrm{oc}}$ after blocking the illumination source (or going from dark to light) is indicative of the photoelectrochemical nature of the material. A photoanode will produce a more negative $E_{\mathrm{oc}}$ under illumination than in the dark, whereas a photocathode will produce a positive $E_{\mathrm{oc}}$ shift under illumination. Thus, a determination can be made quickly for each material by briefly blocking the light source with a shutter and fitting any subsequent change in potential as a linear function (as a simple characterization rather than as a physical model for the decay time profile). A fit with a positive slope indicates a photoanode, one with a negative slope indicates a photocathode, and no change in $E_{\mathrm{oc}}$ suggests that the material is not photoactive.

\section{Conclusions}

Arrays of mixtures of aqueous solutions of metal ions have been quickly and quantitatively deposited onto conductive glass substrates using a commercially available inkjet printer. These metal solutions were then pyrolyzed to form mixed metal oxides. By this method, very large numbers of combinations of up to eight different metals can be formed easily at low cost. We have also developed and demonstrated a screening method to search for materials capable of driving fuel-forming photoelectrochemical reactions. The proof-of-concept experiments presented herein illustrate the speed, flexibility, and ease of use of such systems. Most importantly, the methodology, in which the open-circuit potential of the material is determined, provides critical additional information not readily and rapidly available from photocurrent-only measurements. The approach is easily adaptable to a full array of photoelectrochemical characterization, such as current-voltage behavior, and even the spectral response, flat-band determination by capacitance measurements (Mott-Schottky analysis), and measurement of minority-carrier diffusion lengths.

\section{Acknowledgements}

We acknowledge the Stanford Global Energy and Climate Project, and the U.S. Department of Energy, Office of Basic Energy Sciences, Basic Research for the Hydrogen Fuel Initiative, for support of this work. We also gratefully acknowledge Dr Michael Woodhouse and Prof. Bruce Parkinson at Colorado State University, and Dr Bruce Brunschwig at Caltech, for numerous valuable discussions. T.R.G. acknowledges financial support from the Carl and Shirley Larson SURF Fellowship and from an Amgen Scholars Fellowship.

\section{References}

1 J. M. Bolts and M. S. Wrighton, J. Phys. Chem., 1976, 80, 2641-2645.

2 H. H. Kung, H. S. Jarrett, A. W. Sleight and A. Ferretti, J. Appl. Phys., 1977, 48, 2463-2469.

3 A. Kumar, P. G. Santangelo and N. S. Lewis, J. Phys. Chem., 1992, 96, 834-842.

4 A. J. Nozik, Annu. Rev. Phys. Chem., 1978, 29, 189-222.

5 K. Rajeshwar, P. Singh and J. Dubow, Electrochim. Acta, 1978, 23, 1117-1143.

6 H. O. Finklea, Semiconductor Electrodes, Elsevier, Amsterdam, 1988.

7 A. Heller, Acc. Chem. Res., 1981, 14, 154-162.

8 M. X. Tan, P. E. Laibinis, S. T. Nguyen, J. M. Kesselman, C. E. Stanton and N. S. Lewis, in Progress in Inorganic Chemistry, 1994, vol. 41, pp. 21-144.

9 Z. G. Zou, J. H. Ye, K. Sayama and H. Arakawa, Nature, 2001, 414, 625-627.

10 L. J. Webb, D. J. Michalak, J. S. Biteen, B. S. Brunschwig, A. S. Y. Chan, D. W. Knapp, H. M. Meyer, E. J. Nemanick, M. C. Traub and N. S. Lewis, J. Phys. Chem. B, 2006, 110, 23450-23459.

11 P. T. Hurley, E. J. Nemanick, B. S. Brunschwig and N. S. Lewis, J. Am. Chem. Soc., 2006, 128, 9990-9991.

12 F. R. F. Fan, G. A. Hope and A. J. Bard, J. Electrochem. Soc., 1982, 129, 1647-1649.

13 M. Woodhouse, G. S. Herman and B. A. Parkinson, Chem. Mater., 2005, 17, 4318-4324.

14 T. Arai, Y. Konishi, Y. Iwasaki, H. Sugihara and K. Sayama, J. Comb. Chem., 2007, 9, 574-581.

15 T. F. Jaramillo, S. H. Baeck, A. Kleiman-Shwarsctein, K. S. Choi, G. D. Stucky and E. W. McFarland, J. Comb. Chem., 2005, 7, 264271.

16 S. H. Baeck, T. F. Jaramillo, C. Brandli and E. W. McFarland, J. Comb. Chem., 2002, 4, 563-568.

17 T. F. Jaramillo, A. Ivanovskaya and E. W. McFarland, J. Comb. Chem., 2002, 4, 17-22.

18 L. A. Harris and R. H. Wilson, Annu. Rev. Mater. Sci., 1978, 8, 99134. 